\title{
Effect of Ion Implantation on the Adhesion of Positive Diazoquinone-Novolak Photoresist Films to Single-Crystal Silicon
}

- S. A. Vabishchevich,

- S. D. Brinkevich,

- V.S. Prosolovich,

- N. V. Vabishchevich \&

- D. I. Brinkevich

Journal of Surface Investigation: X-ray, Synchrotron and Neutron Techniques volume 14, pages1352-1357 (2020) Cite this article

- 48 Accesses

- Metricsdetails

Abstract-

The specific energy of detachment of diazoquinone-novolak FP9120 photoresist films of various thicknesses from singlecrystal silicon is studied by the microindentation method. For 5 - $\mu \mathrm{m}$ films, when the normal component of the load is predominant, the values of the specific energy of polymer-film detachment $\mathrm{G}$ are shown to vary within the range 0.1- 0.3 $\mathrm{J} / \mathrm{m}^{2}$. In the case of lateral loading for thin films, the $\mathrm{G}$ value is $3.5-3.7 \mathrm{~J} / \mathrm{m}^{2}$. Implantation with boron and phosphorus ions results in an increase in the $\mathrm{G}$ value. This effect is more pronounced with $\mathrm{P}^{+-i o n}$ implantation. The most probable reason for enhancement of the adhesive interaction at the photoresist- silicon interface is the formation of new chemical bonds between hydroxyl groups on the surface of the oxide layer of the silicon wafer and the photoresist molecules.

This is a preview of subscription content, access via your institution.

\section{REFERENCES}


W. M. Moreau, Semiconductor Lithography. Principles, Practices and Materials (Plenum, New York, 1988; Mir, Moscow, 1990).

2. 2
D. I. Brinkevich, S. D. Brinkevich, N. V. Vabishchevich, V.
B. Odzhaev, and V. S. Prosolovich, Russ.
Microelectron. 43 (3), 194 (2014).

\section{CAS Article Google Scholar}

3. 3

Yu. I. Golovin, Phys. Solid State 50, 2205 (2008).

\section{CAS Article Google Scholar}

4.4

D. I. Brinkevich, V. S. Prosolovich, Yu. N. Yankovskii, S. A. Vabishchevich, N. V. Vabishchevich and V. E. Gaishun, Prib. Metody Izmer. 7 (1), 77 (2016).

\section{Article Google Scholar}

5. 5

L. G. Rosenfeld, J . R. Ritter, T. J . Lardner, and M. R. Lin, J . Appl. Phys. 67 (7), 3291 (1990).

\section{Article Google Scholar}


6. 6

J . Malzbender, J . M. J. Toonder, and A. R. Balkenende, Mater. Sci. Eng., R 36, 47 (2002).

7. 7
S. A. Vabishchevich, N. V. Vabishchevich,
D. I. Brinkevich, V. S. Prosolovich, and S. D. Brinkevich, Vestn. Polotsk. Gos. Univ., Ser. C: Fundam. Nauki, No. 12, 51(2016).

\section{Google Scholar}

8.8
D. I. Tetelbaum, E. V. Kuril'chik, and Yu. A. Mendeleva,
J. Surf. Invest.: X-ray, Synchrotron Neutron Tech. 3, 249 (2009).

\section{Article Google Scholar}

9.9

D. I. Tetelbaum, V. A. Panteleev, A. Yu. Azov, and M. V.

Gutkin, Poverkhn.: Rentgenovskie, Sinkhrotronnye Neitr. Issled., No. 5, 87 (2000).

10. 10

V. L. Levshunova, G. P. Pokhil, and D. I. Tetelbaum,

J. Surf. Invest.: X-ray, Synchrotron Neutron Tech. 5, 276 (2011).

\section{CAS Article Google Scholar}


Experimental Techniques in High Energy

Chemistry, Ed. by M. Ya. Mel'nikov (Izd. Mos. Gos.

Univ., Moscow, 2009) [in Russian].

\section{Google Scholar}

12. 12

Light-Sensitive Polymer Materials, Ed. by A. V. El'tsov (Khimiya, Leningrad, 1985) [in Russian].

\section{Google Scholar}

13. 13

A. A. Kharchenko, D. I. Brinkevich, S. D. Brinkevich, M. G. Lukashevich, and V. B. Odzhaev, J . Surf. Invest.: Xray, Synchrotron Neutron Tech. 9 (1), 87 (2015).

\section{Article Google Scholar}

14. 14

D. I. Brinkevich, A. A. Kharchenko, S. D. Brinkevich, M.

G. Lukashevich, V. B. Odzhaev, V. F. Valeev,

V. I. Nuzhdin, and R. I. Khaibullin, J . Surf. Invest.: X-ray, Synchrotron Neutron Tech. 11(4), 801(2017).

\section{CAS Article Google Scholar}

15. 15 

A. A. Kharchenko, D. I. Brinkevich, S. D. Brinkevich, M.
G. Lukashevich, and V. B. Odzhaev, J . Surf. Invest.: X- ray, Synchrotron Neutron Tech 9 (2), 371 (2015).

\section{Article Google Scholar}

Download references

Author information

Affiliations

1. Polotsk State University, 211440, Novopolotsk, Belarus

S. A. Vabishchevich \& N. V. Vabishchevich

2. Belarusian State University, 220030, Minsk, Belarus

S. D. Brinkevich, V. S. Prosolovich \&D. I. Brinkevich Corresponding authors

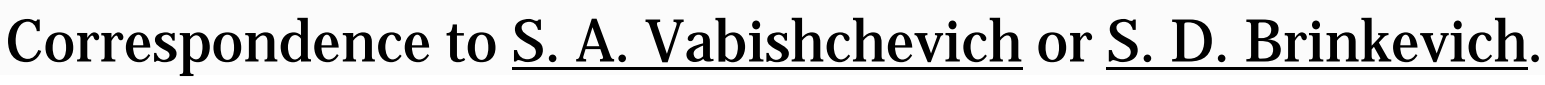
Additional information

Translated by M. Drozdova

Rights and permissions

Reprints and Permissions

About this article

Cite this article

Vabishchevich, S.A., Brinkevich, S.D., Prosolovich, V.S. et al. Effect of Ion Implantation on the Adhesion of Positive Diazoquinone-Novolak Photoresist Films to Single-Crystal Silicon. J. Synch. Investig. 14, 1352-1357 (2020). https://doi.org/10.1134/S1027451020060476 Download citation 
- Received24 December 2019

- Revised20 January 2020

- Accepted25 January 2020

- Published28 December 2020

- Issue DateNovember 2020

- DOlhttps://doi.org/10.1134/S1027451020060476 Keywords:

- diazoquinone-novolak photoresist

- silicon

- adhesion

- ion implantation 